\section{Preferência pela via de parto nas parturientes atendidas em hospital público na cidade de Porto Velho, Rondônia}

\section{Delivery preferences among women giving birth at a public hospital in the city of Porto Velho, in the Brazilian State of Rondônia}

José Ferrari 1

1 Departamento de Medicina. Universidade Federal de Rondônia. Br $364 \mathrm{Km}$ 9,5 sentido Acre. Porto Velho, RO, Brasil. CEP: 76.800-000. E-mail: joferrari10@hotmail.com

\begin{abstract}
Women who come to give birth at the Obstetrics Center of the Hospital de Base in Porto Velho, in the Brazilian State of Rondonia are from underprivileged social groups. Wealthier women attend private clinics and give birth by way of caesarian section at a prescheduled date and time. This article addresses the question of the increase in the incidence of caesarian birth in Latin countries and also in the developed world, where this has provoked necessary and urgent bioethical discussion. It also investigates the opinions of women giving birth at the Obstetrics Center of the Hospital de Base in 2006 and 2007.
\end{abstract}

Key words Cesarean section, Natural childbirth, Personal autonomy

\section{Resumo}

As parturientes que procuram o Centro Obstétrico do Hospital de Base de Porto Velho, Rondônia, para terem seus filhos são mulheres provenientes das camadas sociais desfavorecidas, ao contrário das mulheres com niveis de renda diferenciados que procuram os médicos em seus consultórios particulares e parem seus filhos por meio de cesarianas com dia e hora previamente marcados. $O$ artigo aborda a questão do aumento da crescente incidência de cesarianas verificado em vários países latinos e também no mundo desenvolvido onde tem motivado necessárias e inadiáveis discussões no campo da Bioética e procura conhecer a opinião das parturientes quanto à via de parto que foram atendidas no Centro Obstétrico do Hospital de Base nos anos de 2006 e 2007.

Palavras-chave Cesárea, Parto normal, Autonomia pessoal 


\section{Introdução}

A operação cesariana surgiu como uma necessidade médica. Um recurso salvador para duas vidas, do qual se deve lançar mão para criar uma via de passagem para o feto que, por uma ou outra razão, não consegue ultrapassar as barreiras anatômicas do canal de parto vaginal, dito, por muitos, como sendo natural. E não há como discordar do argumento.

O índice de cesariana nos EUA era de 3\% em 19371 e de 5,5\% em 1970.2 Até então a cirurgia de cesariana era considerada um recurso excepcional destinado a solucionar situações emergenciais em obstetrícia. Tais indicadores se mantiveram estáveis e inferiores a $23 \%$ por mais de 20 anos, chegaram a $27,5 \%$ de cesáreas em 2003. ${ }^{3}$ As taxas de cesarianas se aproximam de $30 \%$, ou seja, índices dez vezes maiores, do que ocorria em 1937,3 ou seja, praticamente uma em cada três gestantes gera seus filhos por meio de incisões abdominais.

Durante o período de sete décadas que separam o ano de 1937 até o ano de 2007, ocorreu significativo incremento na quantidade e na qualidade dos recursos humanos, rotinas de assepsia se tornaram mais rigorosas, as técnicas cirúrgicas se aprimoraram, os riscos anestésicos diminuíram, ocorreu uma melhora no controle das infecções e o importante e decisivo suporte dos bancos de sangue e generalizou-se substancialmente a disponibilidade de medicamentos e antibióticos.

Paralelamente, nas mesmas últimas décadas tem havido incrementos significativos nas taxas de cesarianas em todo o mundo. Países que tradicionalmente apresentavam taxas muito baixas de cesariana, como a Turquia e a Itália, por exemplo, alcançaram, no início dos anos 2000, taxas de 30 e $33 \%$, respectivamente. 4

$\mathrm{Na}$ América Latina, o Chile é o país com a maior taxa de cesariana, com 45\% em 1999, 5 seguido de perto pelo Brasil, que tinha $31 \%$ de cesarianas em 1982 e $37 \%$ de cesarianas em 2000.6

Nas zonas urbanas da China os índices de cesarianas aumentaram de $18,2 \%$ de 1990 até 1992 para $39,5 \%$ de 1998 até 2002.7

Apesar de o Comitê de Aspectos Éticos em Reprodução Humana e Saúde das Mulheres da International Federation of Gynecology and Obstetrics (FIGO) deliberar que é antiético realizar uma cesariana sem indicação médica, ${ }^{8}$ cada vez mais se tem discutido a pertinência ou não da rea-lização de uma cesariana "a pedido".

São fartos e numerosos os textos publicados que investigam ou atribuem ao médico obstetra a culpa pela epidemia de cesarianas chamadas desneces- sárias, 9,10 ou seja, sem uma indicação baseada em evidências científicas. E assim, mulheres querem parir seus filhos por parto normal, mas por influência do médico pré-natalista, acabam mudando de opinião e deixam prevalecer a opinião e a conveniência do obstetra. ${ }^{11}$ Chamam a este modelo como sendo o modelo medicocentrista, tecnológico, no qual o médico, dono de todas as verdades, induz mulheres a fazerem cesarianas sob o fio da lâmina impávida e no ambiente tecnológico e frio dos centros cirúrgicos ao invés de terem partos domiciliares "like" determinados pelas leis da natureza, assim como nascem os cães, os bezerros, os gatos e outros mamíferos.

Quem opta pela via de parto abdominal são mulheres de classe média e alta, presumivelmente mulheres muito mais informadas e esclarecidas do que as mulheres que buscam as maternidades públicas. Seria subestimá-las ao extremo afirmar que optam por cesarianas por pura e simples conveniência do médico que as atendeu durante o pré-natal e as convenceu a optarem pela via de parto abdominal. Principalmente porque se tratam de mulheres informadas e esclarecidas. 12 Além do mais, estaria se fazendo um pré-julgamento de praticamente todos os médicos que atuam na rede privada que tem indicadores de quase $100 \%$ para a via de parto abdominal.

Em Santa Catarina, ao analisar as taxas de cesarianas que subiram de $28,4 \%$ em 2002 para $36,7 \%$ em 2004, Freitas 13 concluiu que "a escolaridade materna, a hora do parto, a presença de patologia e a maior frequência ao pré-natal os fatores que representaram a contribuição mais expressiva" para esse aumento.

Mulheres que se submetem a uma cesariana optam por fazer uma segunda cesariana ou será que o fazem por influência e conveniência do médico que as assiste?

Estudo transversal de Faisal-Cury e Menezes ${ }^{14}$ em clínica privada na cidade de Osasco demonstra claramente que a opção por cesariana está relacionada ao nível de renda - o que já se sabe - e ao grau de satisfação com cesariana prévia.

Analisando 1910 casos de mulheres submetidas a cesariana iterativa no Royal Victoria Hospital da Universidade de Montreal, Gilbert et al. 15 concluíram que o nível educacional das mulheres parece ser determinante na opção por cesariana em mulheres com cesariana prévia.

A opção pela cesariana não é simplesmente uma escolha do médico. É uma escolha da gestante que busca segurança para seu bebê, conforto para si e proteção para a própria pelve. A decisão da gestante 
é baseada em questões intrinsecamente pessoais, como a própria tolerância à dor, sentimentos de alegria, ansiedade, angústia, medo, 16 raça, 17 experiência anterior, 18 grau de aculturação, 19 ocupação, 20 padrão social, ${ }^{21}$ escolaridade, 22 etc.

Lavender et al.23 em uma revisão Cochrane, apontaram a ausência de ensaios clínicos comparando cesariana planejada e parto vaginal planejado e reforçaram a necessidade de revisões sistemáticas de estudos observacionais de boa qualidade." Ou seja, Lavender et al. 23 é conclusivo ao afirmar que os riscos inerentes para cada procedimento ainda carecem de estudos observacionais e que os estudos até agora apresentados, não se apresentam claros.

A Agência Nacional de Saúde Suplementar (ANS) 24 corrobora as mesmas recomendações elencadas pelo Encontro da World Health Organization (WHO) de Fortaleza, em março de 1985, que preconiza índices de cesarianas em torno de $15 \%$ como aceitáveis para qualquer região do planeta. Vale ressaltar que cada um dos eventos está separado por um período de 24 anos no qual ocorreram verdadeiras revoluções na tecnologia e nos meios de comunicação. O conhecimento humano foi democratizado com o recurso da Internet e a informação é simultânea, instantânea em todos os continentes do planeta.

Cyr25 afirma que tentativas para definir um indicador ideal para as cesarianas são fúteis e deveriam ser abandonados. A despeito da opinião de experts de que muitas cesarianas são desnecessárias, os índices continuam a aumentar nos Estados Unidos excedendo $24 \%$ em 2004 - e não há sinais de que venha a decrescer. Realmente, há uma grande discussão em torno de que a aceitação da escolha da paciente pela cesariana eletiva seja uma opção legítima para o nascimento. Cyr cita a frase atribuída a Humpstone: 26

\footnotetext{
"Nós todos nos arrependemos de não ter feito uma cesariana em certos casos, mas não tenho arrependimento de nenhuma das cesarianas que já fiz".
}

No Canadá, onde a incidência de cesarianas aumentou de 5,2\% em 1969 para 25,6 em 2003,27 Armson ${ }^{28}$ alerta que dada a atual incerteza sobre a melhor maneira de nascer, as mulheres que optam por uma cesariana eletiva sem uma indicação médica, aceitam a possibilidade de consequências adversas para elas próprias e seus bebês com o intuito de evitar as incertas e potenciais complicações do parto vaginal.

As mulheres mais idosas preferem cesarianas. ${ }^{29}$
Assim concluiu o trabalho realizado por pesquisadores do Taiwan que investigaram 904.657 nascimentos ocorridos entre os anos de 1997 a 2001 e os mesmos concluem ainda que as gestantes com mais de 34 anos, em especial as primigestas, preferem as cesarianas porque assim fazendo se sentem mais seguras. Um estudo similar ${ }^{30}$ demonstrou que $45,1 \%$ das mulheres chinesas que optam por cesarianas eletivas o fazem por razões astrológicas. A hipótese para a investigação partiu da observação da ocorrência de um elevado número de cesarianas a cada mês de junho, propositadamente antecipadas para que os bebês não nasçam no mês de julho, que é considerado o "mês do fantasma". (the ghost month)

Um estudo ${ }^{31}$ realizado por pesquisadores da Universidade da Carolina do Sul com mulheres não gestantes revelou que as mulheres não brancas têm uma forte preferência por cesarianas e que a ampla maioria das mulheres interrogadas convergiu que a cesariana eletiva é um direito e que deve ter cobertura pelos seguros saúde.

Ao pesquisar o aumento dramático dos índices de cesarianas no sudoeste da China entre os anos de 1994 até 2006 , Zhang et al.32 concluiu que a principal causa para o elevado percentual de cesarianas é o "maternal request", que apresenta variações em função de alguns fatores tais como: nulipararidade, nível de escolaridade elevada, gestantes idosas, acesso a determinados hospitais e o tipo de ocupação da gestante.

Faundes et al. 33 conclui em 2004 que o conceito de que a principal causa na taxa de cesárea é o respeito dos desejos das mulheres por parte dos médicos não tem sustentação na opinião declarada pelas mulheres. Uma melhor comunicação entre médicos e mulheres grávidas talvez possa contribuir para melhoria da situação atual. É de se presumir que melhorar a situação atual é sinônimo de reduzir as taxas de cesariana a pedido.

O desejo de "eu mesma empurrar o bebê para fora" parece ser determinante naquelas mulheres que optam pelo parto vaginal e, dentre destas, o relato de incontinência urinária ou anal no passado não foi determinante na preferência pela via de parto vaginal neste grupo de mulheres, 34 muito embora puérperas americanas alegam que desejariam ter recebido mais informações sobre incontinência urinária ou anal e que isso poderia ter influenciado a escolha da via parto. 35,36

No Bronx, bairro de New York, a ampla maioria das mulheres entrevistadas 37 acredita que a cesariana a pedido não é um procedimento seguro, oportuno, prudente (advisable), no entanto em Hong Kong é muito frequente que primigestas submetidas a parto 
normal mudem a preferência para a via de parto abdominal em nova gestação, especialmente diante de intercorrências ou complicações durante o primeiro nascimento. 38

O objetivo do presente trabalho é conhecer a preferência pela via de parto entre as mulheres que tiveram filhos no Centro Obstétrico do Hospital de Base de Porto Velho entre os anos de 2006 e 2007 e assim, contribuir para uma reflexão sobre a emblemática questão das cesarianas, cujos índices insistem em continuar ascendendo, a despeito das campanhas em defesa do parto normal.

\section{Métodos}

Foi realizado um estudo transversal, documental, em uma unidade pública da cidade de Porto Velho, capital do Estado de Rondônia: o Hospital de Base Ary Tupinambá Pena Pinheiro, conhecido como Hospital de Base.

O Hospital de Base serve como referência para o atendimento terciário, não somente para a capital, mas para todo o Estado de Rondônia.

Foi realizado um inquérito em todos os prontuários médicos levantados e relacionados aos nascimentos ocorridos durante os anos de 2006 e 2007 e, destes, coletados dados sumários sobre as gestantes, tais como identificação, idade, endereço, tipo de parto, dados da história obstétrica e dados relacionados ao procedimento realizado.

O projeto foi aprovado no Comitê de Ética em Pesquisa da Universidade Federal de Rondônia no parecer 4433.0.000.047-08.

A coleta dos dados foi feita a partir de um instrumento padronizado e pré-codificado com especificidade para a pesquisa.

O questionário contendo 76 perguntas foi dividido em três partes: a primeira com 29 perguntas relacionadas aos dados sociais e demográficos das gestantes; a segunda com 27 perguntas relacionadas à história reprodutiva e a terceira, com 20 perguntas relacionadas à experiência do nascimento propriamente dito.

As perguntas do questionário foram dirigidas às gestantes que foram procuradas e entrevistadas em suas residências na conformidade dos endereços coletados no prontuário do Centro Obstétrico.

A amostra foi constituída por todas as parturientes $(n=4710)$ atendidas no Centro Obstétrico do Hospital de Base nos anos de 2006 e 2007. Foram excluídas da amostra as mulheres cujas residências se situavam na zona rural - de difícil acesso - ou em outros municípios do Estado.

As entrevistas foram realizadas por equipe composta por profissionais da área da saúde, agentes de saúde, previamente treinados e supervisionados por este pesquisador que se deslocaram à residência das entrevistadas. Quando as mulheres não eram encontradas no endereço especificado no prontuário ou o próprio endereço não existia - situação bastante freqüente - eram excluídas.

Todos os questionários foram revisados e os dados armazenados no programa Statistical Package for the Social Sciences (SPSS) 15D. As dúvidas surgidas foram ratificadas por telefone ou com retorno à moradia de cada entrevistada.

Os dados foram coletados após a assinatura do Termo de Consentimento Livre e Esclarecido.

\section{Resultados}

A Tabela 1 registra a via de parto com distribuição das gestantes por faixa etária, verificando-se que ocorre praticamente a mesma proporção entre as vias de parto nas três faixas. Quando se analisa a totalidade da amostra, observa-se uma distribuição praticamente igual para ambas as vias de parto

Na Tabela 2 são analisados os óbitos fetais ocorridos no período considerado e o teste de Pearson aponta que não existe relação entre óbitos fetais e a via de parto escolhida para as parturientes atendidas.

Durante a realização do questionário, foi perguntado às mulheres se era seu desejo ter seus filhos pela via vaginal e 857 (68\%) das mulheres responderam que esta era a via de parto desejada (Tabela 3). Dentre estas, 348 (40,6\%) tiveram indicação para realização de cirurgia cesariana e havendo a episiotomia em quase $3 \%$ dos casos.

Os dados da Tabela 4 corroboram o desejo das mulheres de baixa renda pelo parto vaginal independentemente do nível de escolaridade declarado.

Quando perguntadas qual via de parto desejariam em gestação futura (Tabela 5), se verifica que as mulheres submetidas a parto normal $(n=625)$ têm uma clara preferência $(85,3 \%)$ pela mesma via de parto, enquanto $41 \%$ das mulheres submetidas à cesarianas mantêm a preferência pela mesma via de parto em futuras gestações $(p<0,001)$.

Quando perguntadas sobre o tempo de recuperação para cada uma das vias de parto, as opiniões foram claramente convergentes e a ampla maioria das mulheres respondeu que o tempo de recuperação da cesariana é maior.

Quando perguntadas sobre se "independentemente das condições financeiras, a gestante tem o direito de escolher o tipo de parto a ser realizado", $90,8 \%$ responderam que sim, $8,1 \%$ responderam que não e $1,1 \%$ não souberam responder. 
Tabela 1

Via de parto das parturientes atendidas no Centro Obstétrico do Hospital de Base nos anos de 2006 e 2007 conforme faixa etária.

\begin{tabular}{|c|c|c|c|c|c|c|c|c|}
\hline \multirow[t]{3}{*}{ Via de parto } & \multicolumn{6}{|c|}{ Faixa etária (anos) } & \multicolumn{2}{|c|}{ Total } \\
\hline & \multicolumn{2}{|c|}{ até 19} & \multicolumn{2}{|c|}{ De 20 até 35} & \multicolumn{2}{|c|}{36 ou mais } & \multirow[b]{2}{*}{$\mathrm{n}$} & \multirow[b]{2}{*}{$\%$} \\
\hline & $\mathrm{n}$ & $\%$ & $\mathrm{n}$ & $\%$ & $\mathrm{n}$ & $\%$ & & \\
\hline Vaginal & 206 & 48,8 & 395 & 50,3 & 24 & 44,4 & 625 & 49,5 \\
\hline Cesariana & 216 & 51,2 & 390 & 49,7 & 30 & 55,6 & 636 & 50,5 \\
\hline Total & 422 & 100,0 & 785 & 100,0 & 54 & 100,0 & 1261 & 100,0 \\
\hline
\end{tabular}

Fonte: SAME/Hospital de Base.

Tabela 2

Óbitos fetais ocorridos e a via de parto das parturientes atendidas no Centro Obstétrico do Hospital de Base nos anos de 2006 e 2007.

\begin{tabular}{llrrrrr}
\hline Via de parto & \multicolumn{4}{c}{ Óbito fetal } & \multicolumn{2}{c}{ Total } \\
\cline { 2 - 6 } & \multicolumn{2}{c}{ Sim } & \multicolumn{3}{c}{ Não } \\
\cline { 2 - 6 } & $\mathrm{n}$ & $\%$ & $\mathrm{n}$ & $\%$ & $\mathrm{n}$ & $\%$ \\
\hline Vaginal & 14 & 2,3 & 611 & 97,8 & 625 & 100,0 \\
Cesariana & 22 & 3,5 & 614 & 96,5 & 636 & 100,0 \\
Total & 36 & 2,9 & 1225 & 97,1 & 1261 & 100,0 \\
\hline
\end{tabular}

Fonte: SAME/Hospital de Base. $\left(\chi^{2}=1,689 ; p=0,194\right)$.

Tabela 3

Desejo das parturientes atendidas nos Centro Obstétrico do Hospital de Base nos anos de 2006 e 2007 em relação à via de parto.

\begin{tabular}{lrr}
\hline Desejavam ter parto normal & N & $\%$ \\
\hline Sim & 857 & 68,0 \\
Não & 367 & 29,1 \\
Não souberam responder & 37 & 2,9 \\
Total & 1261 & 100,0 \\
\hline
\end{tabular}

Fonte: SAME/Hospital de Base. 
Desejo das parturientes atendidas nos Centro Obstétrico do Hospital de Base nos anos de 2006 e 2007 em relação à via de parto cesariana e escolaridade.

\begin{tabular}{|c|c|c|c|c|c|c|}
\hline \multirow[t]{3}{*}{ Escolaridade } & \multicolumn{4}{|c|}{ Desejo ter cesariana } & \multicolumn{2}{|c|}{ Total } \\
\hline & \multicolumn{2}{|c|}{ Sim } & \multicolumn{2}{|c|}{ Não } & \multirow[b]{2}{*}{$\mathrm{n}$} & \multirow[b]{2}{*}{$\%$} \\
\hline & $\mathrm{n}$ & $\%$ & $\mathrm{n}$ & $\%$ & & \\
\hline Analfabeta & 1 & 4,5 & 21 & 95,5 & 22 & 100,0 \\
\hline Fundamental incompleto & 169 & 20,5 & 309 & 79,5 & 478 & 100,0 \\
\hline Fundamental completo & 44 & 26,8 & 120 & 73,2 & 164 & 100,0 \\
\hline Médio incompleto & 61 & 29,5 & 146 & 70,5 & 207 & 100,0 \\
\hline Médio completo & 93 & 29,4 & 224 & 70,6 & 317 & 100,0 \\
\hline Superior incompleto & 7 & 22,6 & 22 & 77,4 & 31 & 100,0 \\
\hline Superior completo & 1 & 14,3 & 6 & 85,7 & 7 & 100,0 \\
\hline
\end{tabular}

Fonte: SAME/Hospital de Base.

\section{Tabela 5}

\begin{tabular}{|c|c|c|c|c|c|c|}
\hline \multirow[t]{3}{*}{ Via de parto } & \multicolumn{4}{|c|}{ Via de parto desejada em futura gestação } & \multicolumn{2}{|c|}{ Total } \\
\hline & \multicolumn{2}{|c|}{ Vaginal } & \multicolumn{2}{|c|}{ Cesariana } & \multirow[b]{2}{*}{$\mathrm{n}$} & \multirow[b]{2}{*}{$\%$} \\
\hline & $\mathrm{n}$ & $\%$ & $\mathrm{n}$ & $\%$ & & \\
\hline Vaginal & 533 & 85,3 & 92 & 14,7 & 625 & 49,5 \\
\hline Cesariana & 375 & 59,0 & 261 & 41,0 & 636 & 50,5 \\
\hline Total & 908 & 72,0 & 353 & 28,0 & 1261 & 100,0 \\
\hline
\end{tabular}

Fonte: SAME/Hospital de Base. $\left(\chi^{2}=108,31 ; p<0,001\right)$.

\section{Discussão}

$\mathrm{O}$ índice de 50,4\% de cesarianas registrado no Centro Obstétrico do Hospital de Base nos anos de 2006 e 2007, considerado elevado para os padrões estabelecidos, pode se justificar pelo fato que em junho de 2006 foi inaugurada a Maternidade Municipal e, a partir de então, o Hospital de Base passou a ser referência para gestações de alto risco. 39

Na Coréia do Sul, onde os índices de cesarianas são considerados epidêmicos ( $40 \%$ no ano 2000$)$, um inquérito conduzido 40 a 505 mulheres entre 20 a 49 anos revelou que $95 \%$ das mulheres preferiam a via de parto vaginal e aconselhariam outras mulheres a terem seus filhos por esta via.

$\mathrm{Na}$ Itália, onde as taxas de cesarianas atingiram $33,2 \%$ no ano de 2000 , um trabalho similar 4 revelou que $93 \%$ das mulheres italianas que tiveram partos normais preferem a via vaginal e $73 \%$ das cesariadas preferem a Via Abdominal para terem seus filhos.

No Chile, onde as taxas de cesarianas estavam em torno de $40 \%$ em $1997,77,8 \%$ das mulheres submetidas a questionário estruturado, declararam que preferiam a via de parto vaginal, 9,4\% declararam preferir a cesariana e $12,8 \%$ declararam não ter qualquer preferência. 41

Em Singapura, um estudo do mesmo gênero, demonstrou que a ampla maioria das mulheres asiáticas preferem a via vaginal, no entanto as demandas por cesarianas nos serviços de obstetrícia se assemelham a dos países ocidentais. 42

$\mathrm{Na}$ Suécia, onde os índices de cesarianas permanece em torno de 10 a $12 \%$ durante mais de 20 anos, estes índices atingiram taxas de $20 \%$ em 1999. Verificou-se que as mulheres que preferem cesarianas tem nível de escolaridade mais alto, são 
urbanas, tem história obstétrica prévia negativa e são mais preocupadas com as consequências e riscos do nascimento. 43

Assim sendo, se a absoluta maioria das mulheres deseja ter filhos através de parto normal, pode-se perguntar: Por que as taxas de cesarianas apresentam indicadores crescentes e sem qualquer perspectiva de declínio? É possível que a resposta esteja ligada ao fato de que no Brasil não existe uma "cultura" feminina que valorize a cesariana como preferência. 44

Neste sentido também se encontra no Datasus 45 a informação que no ano de 2006, o Estado de Rondônia registrou 24.932 nascidos vivos, sendo que na capital Porto Velho ocorreu o nascimento de 7637 nascidos vivos, sendo que 3991 (52,25\%) foram por via vaginal e 3619 (47,9\%) através de cesarianas e outros 37 ignorados. Do total de 7637 nascimentos, aproximadamente $4500(\sim 60 \%)$ ocorreram na rede pública, que tem como referência o Hospital de Base e a Maternidade Municipal Mãe Esperança, e aproximadamente $40 \%$ dos nascimentos ocorreram na rede suplementar. Números e indicadores parecidos se repetem no ano de 2007. Desse modo pode-se perguntar também: Por que $40 \%$ das gestantes têm seus filhos com médicos e em hospitais particulares? A partir dos presentes dados alguns aspectos podem ser levados em conta.

As mulheres atendidas no Centro Obstétrico do Hospital de Base, que é uma instituição pública, são, em absoluta maioria, provenientes das camadas populacionais de baixa renda. Todas passaram pela experiência de já terem tido pelo menos uma gestação e os nascimentos ocorreram nos anos de 2006 e 2007 na instituição citada. Ao serem perguntadas sobre a preferência pela via de parto antes daquela gestação $68 \%$ (Tabela 3 ) responderam que desejavam, àquela ocasião, terem seus filhos por via vaginal e $72 \%$ declararam que, em futura gestação, desejarão ter seus filhos pela mesma via. Ademais há uma clara relação entre a via de parto escolhida e a experiência anterior (Tabela 5).

Por outro lado fato conhecido também que, no Brasil, as gestantes de baixa renda fazem o pré-natal nos postos de saúde e são, geralmente, atendidas por enfermeiras que seguem as rotinas de programas préestabelecidos pelo Ministério da Saúde. As consultas são agendadas para determinados dias da semana. Assim quando é chegado o momento do nascimento, a gestante é encaminhada para o hospital ou maternidade e é atendida por pessoas desconhecidas, plantonistas, profissionais com os quais não tem qualquer vínculo, com os quais não estabeleceu nenhuma relação de confiança. Neste contexto, é possível que, em algumas ocasiões, se estabeleçam vínculos entre a gestante e a enfermeira que realiza o acompanhamento pré-natal, mas, definitivamente, não se estabelecem vínculos com o sistema. $\mathrm{O}$ atendimento é, mormente, impessoal. A insegurança e o medo são consequências que se multiplicam nas primigestas.

Os dados obtidos com as gestantes atendidas no Centro Obstétrico do Hospital de Base sobre a preferência pela Via de parto são concordantes com a literatura, no sentido de que a ampla maioria das mulheres manifesta o desejo de terem seus filhos por meio de parto normal. Da mesma forma que opinam favoravelmente pelo direito de opção pela cesariana. A indicação da via de parto deveria, portanto, levar em conta a satisfação da mulher grávida, seus anseios, seus medos e seus desejos e não as decisões burocráticas que, baseados em prerrogativas pouco consistentes, se propõem a tutelar a vontade das usuárias do sistema de saúde que carregam seres humanos em formação no interior de seus úteros e muitos outros tantos projetos e planos de vida em seus sonhos. Dentro do espírito da ética kantiana de "ter a coragem de ser livre e respeitar a liberdade dos outros", declara o Datasus: 45

\footnotetext{
"Nenhuma liberdade é livre se não se respeitam, em conjunto, essas liberdades, seja qual for sua forma de governo; e nenhuma sociedade é completamente livre se tais liberdades não existirem em caráter absoluto e sem reservas. A única liberdade merecedora desse nome é a de buscar nosso próprio bem da maneira que nos seja conveniente, contanto que não tentemos privar outros do que lhes convém, ou impedir seus esforços de obtê-lo. Cada um é o guardião adequado de sua própria saúde, seja física, seja mental ou espiritual. A liberdade ganha mais tolerando que cada um viva conforme o que lhe parece bom do que compelindo cada um a viver conforme pareça bom ao restante". 46
}

Em março de 2006, o National Institute of Health, na State of the Science Conference47 concluiu que:

- não existem evidências científicas suficientes para comprovar amplamente os benefícios da cesariana eletiva em relação ao parto normal;

- até que existam evidências suficientes, os casos de cesariana a pedido devem ser considerados cuidadosamente e individualizados de acordo com os princípios éticos;

- dados os riscos aumentados de placenta prévia ou acreta nas mulheres com cesariana prévia, a cesariana eletiva não deve ser recomendada em mulheres que desejem ter muitos filhos;

- as cesarianas eletivas não devem ser realizadas 
antes de 39 semanas ou sem a verificação da maturidade pulmonar do neonato para se evitar complicações respiratórias.

\section{Referências}

1. Duncan CJ, Doyle JB. Cesarean section: a ten year study of 703 cases at the Boston City Hospital. NEJM. 1937; 216: 15.

2. Centers for Disease Control and Prevention. Rates of cesarean delivery - United States, 1991. Morb Mortal Wkly Rep. 1993; 42: 285-9.

3. Hamilton BE, Martin JA, Ventura, SJ. Births: preliminary data for 2005. Health e-stats. Hyattsville, MD; National Center for Health Statistics, November 21, 2006.

4. Donati S, Gandolfo ME, Andreozzi S. Do Italian mothers prefer cesarean delivery? Birth. 2003; 30: 89-93.

5. Murray SF, Serani F. Recent trends in cesarean sections rates in Chile - the political economy of maternal care. Santiago de Chile: Instituto Nacional de Estadísticas; 2000. Available in: http://www.ine.cl/chile-cifras.

6. IBGE (Instituto Brasileiro de Geografia e Estatística) Matéria publicada na Folha de São Paulo. [Acesso em 11 jan 2006]. Disponível em: www.ans.gov.br/portal/site/ home2/destaque_22585_2.asp?secao=Home.

7. Tang $\mathrm{S}, \mathrm{Li} \mathrm{X}, \mathrm{Wu} \mathrm{Z}$. Rising cesarean delivery rate in primiparous women in urban China: evidence from three nationwide household health surveys. Am J Obst Gynecol. 2006; 195: $1527-32$

8. Schenker JG, Cain JM. FIGO Committee Report. FIGO Committee for the Ethical Aspects of Human Reproduction and Woman's Health. International Federation of Gynecology and Obstetrics. In J Gynaecol Obstet. 1999; 64: 317-22.

9. Potter JE, Hopkins K, Faundes A, Perpétuo I. Women's autonomy and scheduled cesarean sections in Brazil: a cautionary tale. Birth. 2008; 35: 33-40.

10. Souza MR. Parto: entre o desejo e a realização. Trabalho apresentado no XIII Encontro Brasileiro de Estudos Populacionais em Ouro Preto, Minas Gerais, Brasil, de 4 a 8 de novembro de 2002. A pesquisa foi coordenada pela Dra. Elza Berquó e contou com financiamento do Fundo de Populações das Nações Unidas (FNUAP) e National Institute od Health (NIH). [Acesso em 16 jul 2009]. Disponível em: http://www.pbh.gov.br/smsa/bhpelopartonormal/estudos_cientificos/arquivos/parto_entre_o_desejo_ e_a_realizacao.pdf.

11. Gamble JA, Creedy DK. Women's request for a cesarean section: a critique of the literature. Birth. 2000; 27: 256-63.

12. Fabri RH, Lima e Silva HS, Lima RV, Murta EFC. Estudo comparativo de cesariana entre um hospital público universitário e um hospital privado. Rev Bras Saúde Matern Infant. 2002; 2: 29-35.

13. Freitas PF, Sakae TM, Jacomino MEMLP, Polli L. Fatores médicos e não-médicos associados às taxas de cesariana em um hospital universitário no Sul do Brasil. Cad Saúde Pública. 2008; 24: 1051-61.
Há que se respeitar a autonomia e o livre arbítrio que é dado a cada pessoa humana para decidir sobre o que fazer com seu próprio corpo.

14. Faisal-Cury A, Menezes PR. Fatores associados à preferência por cesariana. Rev Saúde Pública. 2006; 40: 226-32.

15. Gilbert A, Fraser W, Benjamin A, Abenhaim H. Does education level influence request for elective repeat cesarean delivery among women with a previous cesarean delivery? Am J Obstet Gynecol. 2008; 199 (Suppl. 1): S107.

16. McCourt C, Weaver J, Statham H, Beake S, Gamble J, Creedy DK. Elective cesarean section and decision making: a critical review of the literature. Birth. 2007; 34: 65-79.

17. Kabir AA, Pridjian G, Steinmann WC, Herrera EA, Khan MM. Racial differences in cesareans: an analysis of US 2001 National Inpatient Sample Data. Obstet Gynecol. 2005; 105: 710-8.

18. Pare E, Stamilio D, Cahil A, Stevens E, Peipert J, Macones G. Maternal morbidity with elective repeat cesarean section: the influence of the number of previous cesarean sections. Am J Obstet Gynecol. 2004; 191 (Suppl. 1): S153.

19. Zlot AI, Jackson DJ, Korenbrot C. Association of acculturation with cesarean section among Latinas. Matern Child Health J. 2005; 9: 11-20

20. Simoes E, Kunz S, Bosing-Schwenkglends M, Schmahl. Occupation and risk of cesarean section: study based on the perinatal survey of Baden-Württemberg, Germany. Arch Gynecol Obstet. 2005; 271: 338-42.

21. Yazlle MEHS, Rocha JSY, Mendes MC, Patta MC, Marcolin AC, Azevedo GD. Incidência de cesáreas segundo financiamento da assistência ao parto. Rev Saúde Pública. 2001; 35: 202-6.

22. Knup VMA, Melo ECP, Oliveira RB. Distribuição do parto vaginal e da cesariana no município do Rio de Janeiro no período de 2001 a 2004. Rev Enferm. 2008; 12: 39-44.

23. Lavender T, Hofmeyer GJ, Neilson JP, Kingdon C, Gyte GMI. Caesarean section for non-medical reasons. Cochrane Database of Systematic Reviews, Issue 3. Art. $\mathrm{n}^{\mathrm{o}}$ : CD0046660. DOI:10.1002/14651858. CD004660. Pub2, 2006

24. Brasil. Agência Nacional de Saúde Suplementar. O modelo de atenção obstétrica no setor de saúde suplementar no Brasil: cenários e perspectivas. Rio de Janeiro; 2008. [Acesso em 21 abr 2008]. Disponível em: http://www.ans.gov.br/portal/upload/noticias/clipping/livro parto_web.pdf.

25. Cyr RM. Myth of the ideal cesarean section rate. Commentary and historic perspective. Am J Obstet Gynecol. 2006; 194: 932-6.

26. Humpstone OP. Cesarean section vs spontaneous delivery. Am J Obstet Gynecol. 1920; 1: 986-9, 994-6.

27. Nair C. Trends in cesarean deliveires in Canadá. Health Reprod. 1991; 3: 203-19. 
28. Armson BA. Is planned cesarean childbirth a safe alternative? CMAJ. 2007; 176: 475-6.

29. Lin HC, Sudha Xirasaghar MBBS. Maternal age and the likelihood of a maternal request for cesarean delivery: a 5year population-based study. Am J Obstet Gynecol. 2005; 192: 848-55.

30. Huang CY, Yang MC, Chen WC. Maternal factors associates with use of cesarean section: a case study of the National University Hospital (in Chinese). J Public Health. 1997; 16: 309-18. [Acesso em 27 jul 2009] .Disponível em: http://pt.wkhealth.com/pt/re/jech/abstract.

31. Thurman AR, Zoller JS, Swift SE. Non-pregnant patient's preference for delivery route. Int Urogynecol J. 2004; 15: 308-12.

32. Zhang J, Meikle S, Zheng J, Sun W. Li Z. Cesarean delivery on maternal request in southeast China. Obstetric Gynecol. 2008; 111: 1077-82.

33. Faundes A, Karla Simônia de Pádua KS, Osis MJD, Cecatti JG, Sousa MH. Opinião de mulheres e médicos brasileiros sobre a preferência pela via de parto. Rev Saúde Pública. 2004; 38: 488-94.

34. Tedesco RP, Maia Filho NL, Mathias L, Benez AL, Castro VCL, Bourroul GM, Reis FI. Fatores determinantes para as expectativas de primigestas acerca da Via de Parto. Rev Bras Ginecol Obstet. 2004; 26: 791-8.

35. Kudish B, McNeeley G, Hendrix S. Factors affecting patient preferences for mode of delivery. Am J Obstet Gynecol. 2007; 197 (Suppl. 1): S78.

36. Renner RM, Eden KB, Osterweil P, Chan BK, Guise JM. Informational factors influencing patient's childbirth preferences after prior cesarean. Am J Obstet Gynecol. 2007; 196: e14-6.

37. Pevzner L, Preslicka V, Bush M, Chan K. Patient's attitudes associated with cesarean delivery on maternal request in a urban population. Am J Obstet Gynecol. 2008; 198: e35-7.

38. Prefeitura do Município de Porto Velho. [ acesso em $13 \mathrm{dez}$ 2010]. Disponível em: http://www.portovelho.ro.gov.br/ index.php?option $=$ com content $\&$ task $=$ view $\&$ id $=328 \&$ Item $\mathrm{id}=178$.
39. Pang MW, Leung TN, Lau TK, Chung TKH. Impact of first childbirth on changes in women's preference for mode of delivery: follow-up of a longitudinal observational study. Birth. 2008; 35: 121-8.

40. Sang-II L, Khang Y, Lee MS. Women's attitudes toward mode of delivery in South Korea - a society with high cesarean section Rates. Birth. 2004; 31: 108-16.

41. Angeja ACE, Whashington AE, Vargas JE, Gomes R, Rojas I, Caughey AB. Chilean women's preferences regarding mode of delivey: wich do they prefer and why? BJOG. 2006; 113: 1253-8.

42. Chong ESY, Monguelli M. Attitudes of Singapore women toward cesarean and vaginal deliveries. Int $\mathrm{J}$ Gynacol Obstet. 2003; 80: 189-94.

43. Hildingsson I, Radestad I, Rubertson C, Waldestron U. Few woman wish to be delivered by cesarean section. BJOG. 2002; 109: 618-23.

44. Barbosa GP, Giffin K, Ângulo-Tuesta A, Gama AS, Chor D, D'Orsi E, Reis ACGV. Parto cesáreo: quem o deseja? Em quais circunstâncias? Cad Saúde Pública. 2003; 19: 1611-20.

45. Brasil. Ministério da Saúde. Datasus - Informações de Saúde. [acesso em $28 \mathrm{dez}$ 2009]. Disponível em: http://tabnet.datasus.gov.br/cgi/deftohtm.exe?sinasc/cnv/nv ro.def .

46. Mill JS. A liberdade e utilitarismo. São Paulo: Editora Martins Fontes; 2000. p. 22.

47. National Institute of Health. State-of-the-Science Conference. March 27-29, 2006. Cesarean delivery on maternal request: final statement. Obstet Gynecol 2006; 23 : 1386-97. [acesso em $29 \mathrm{dez}$ 2009]. Disponível em: http://consensus.nih.gov/2006/2006CesareanSOS027main. htm

Recebido em 5 de julho de 2010

Versão final apresentada em 26 de outubro de 2010

Aprovado em 30 de novembro de 2010 